\title{
"CHARACTERISING THE ELECTRON DENSITY FLUCTUATIONS IN THE HIGH-LATITUDE IONOSPHERE AT SWARM ALTITUDE IN RESPONSE TO THE GEOMAGNETIC ACTIVITY,
}

\author{
Fabio Giannattasio ${ }^{*}, 1$, Paola De Michelis ${ }^{1}$, Giuseppe Consolini², \\ Virgilio Quattrociocchi ${ }^{2}$, Igino Coco ${ }^{1}$ and Roberta Tozzi ${ }^{1}$ \\ (1) Istituto Nazionale di Geofisica e Vulcanologia, Rome, Italy \\ (2) INAF-Istituto di Astrofisica e Planetologia Spaziali, Rome, Italy
}

Article history

Receveid May 31, 2018; accepted September 24, 2018.

Subject classification:

Electron density fluctuations; Ionosphere; Swarm; Geomagnetic activity; Space Weather

\begin{abstract}
The high-latitude ionosphere is characterised by plasma density irregularities with typical lengths in a wide range of scales (from $\sim 1 \mathrm{~m}$ up to $\sim 1000 \mathrm{~km}$ ). The enhancement of these irregularities caused for instance by severe Space Weather conditions can affect trans-ionospheric communications between ground facilities and satellites. For this reason, an accurate characterisation of the dynamic properties of electron density and their variation with the geomagnetic activity level is of particular interest for the Space Weather especially at high latitudes. In this framework, taking advantage of high resolution in situ measurements by the recent ESA-Swarm space mission orbiting in the ionospheric F-layer, we study both the dynamical properties of the electron density and the scaling properties of the electron density fluctuations at high latitudes in the Northern and Southern Hemispheres in response to changes in the geomagnetic activity levels via nonlinear techniques involving the first-order structure functions. Indeed, it has been shown that the turbulent character of the ionospheric plasma density plays an important role in the generation and dynamics of ionospheric plasma density irregularities and the study of the scaling properties of the electron density fluctuations permits us to characterise the possible turbulent state of the ionospheric electron density. The obtained results are consistent with the turbulent character of the ionospheric dynamics, and with the presence of different turbulent regimes that show a dependence on the geomagnetic activity levels, magnetic latitude and MLT values.
\end{abstract}

\section{INTRODUCTION}

In the last years the dependence of our society on the Global Navigation Satellite System (GNSS) has increased substantially. Critical applications, such as railway control, highway traffic management, precision agriculture, emergency response, commercial aviation, and marine navigation, require and depend on GNSS services. The accurate timing provided by Global Positioning System (GPS), which is just one component of
GNSS, facilitates everyday activities, such as banking, mobile phone operations, and even the control of power grids. As our national critical infrastructures and economy are increasingly dependent on positioning, navigation, and timing services, our society is vulnerable to damages that can be caused by Space Weather on space-borne and ground-based technological systems. Nowadays, it is understood that Space Weather is the largest contributor to single-frequency GPS errors and a significant factor for differential GPS. Indeed, GPS re- 
ceivers calculate their locations by analysing signals from a constellation of satellites, but these signals can be delayed or distorted while passing through the ionosphere. Consequently, Space Weather phenomena, such as solar flares and geomagnetic storms, can result in errors in position and navigation, and degradation or loss of signals.

In order to try to solve this problem, in the last years a great attention has been devoted to the study of the spatial and temporal characteristics of the ionosphere all over the globe and of its global and local phenomena. It was found that the high-latitude ionosphere, which is characterised by a strong coupling between the magnetosphere and ionosphere through electric fields and currents as well as particle flows, hosts plasma density irregularities from scale lengths of $1000 \mathrm{~km}$ down to a few meters [e.g., Keskinen and Ossakow, 1983; Tsunoda, 1988; Oksavik et al., 2012] and a close relationship exists between these irregular structures and GPS signal failures [Prikryl et al., 2011; Yaqi et al., 2014; Jin et al., 2015]. Indeed, the ionospheric irregularities such as polar patches and auroral blobs at high-latitude, but also the equatorial ionization anomaly and the equatorial plasma irregularities at low-latitude, can influence the quality of GNSS signal being responsible of the well-known scintillation phenomena on GNSS. Recently, Xiong et al. [2016, 2018] found that the large density gradients associated with ionospheric plasma irregularities are a crucial factor for causing the GPS signal loss of Swarm satellites. Several other studies in literature have pointed out that ionospheric turbulence is of central importance in driving these irregularities, being capable of generating coherent structures and plasma inhomogeneities [see, e.g., Basu et al. 1984; Basu et al. 1988; Earle et al. 1989]. In fact, in situ measurements below $1000 \mathrm{~km}$ altitude have established that the one-dimensional spectra of the density irregularities obey power-law scaling suggesting the existence of turbulence phenomena. However, the origin of the irregularities is a complex process, which can only partly be associated with turbulent processes that are not easy to discern. It has been suggested by Basu et al. [1984], for example, that velocity shear driven turbulence is capable of enhancing the irregularities at short scale lengths and both satellite and rocket experiments have indicated the existence of a correlation between electrostatic turbulence and sheared crossed-field plasma flow arising from inhomogeneous electric fields [e.g., Basu et al., 1988; Earle et al., 1989]. Thus, the global changes of the solar windmagnetosphere-ionosphere conditions can play a fundamental role in affecting the global ionospheric dynamics and in driving turbulent phenomena.
In light of these studies, the characterization of the ionospheric turbulence at Swarm altitude is of great importance to provide information on the mechanisms responsible for the generation and dynamics of ionospheric inhomogeneties or plasma density irregularities. In this framework, statistical studies of the average pattern of magnetic field and plasma parameters as a function of the different geomagnetic conditions can allow us to construct dynamic models of the ionospheric state and to unveil the underlying multiscale nature of its dynamics. The recent ESA-Swarm space mission [Friis-Christensen et al., 2006], which consists of three satellites (Alpha, Bravo, and Charlie) placed in two different polar orbits; Alpha and Charlie fly side by side at an altitude that initially was of about $470 \mathrm{~km}$, Bravo flies at an altitude that initially was of about 520 $\mathrm{km}$, provides a unique opportunity to make studies on the magnetic field and plasma parameter conditions in the ionosphere.

The purpose of this work is to characterize, from a statistical point of view, the scaling properties of the electron density at the average altitude of $\sim 460 \mathrm{~km}$, in order to gain new insights on the ionospheric turbulence in response to different conditions of the geomagnetic activity level. In particular, the ionospheric electron density is put in relation with the different quiet/disturbed conditions of the magnetosphere due to the interaction between the geomagnetic field and the plasma of solar origin in order to shed light on the nonlinear coupling between solar wind, magnetosphere and ionosphere. This kind of research can be done using data recorded by one of the three Swarm satellites (Swarm A) for a period of two years (1 April 2014 to 31 March 2016). The amount of data available is in fact sufficient to guarantee that the obtained results are statistically significant.

The paper is organised as follows. In Sections 2 and 3 we describe the data set used and the analysis performed; in Section 4 we show the obtained results and discuss them; in Section 5 we drive conclusions and highlight the future perspectives.

\section{SWARM DATA AND THEIR PROCESSING}

The data set used in this work was acquired by the Swarm A satellite [Friis-Christensen et al., 2006] from 1 April 2014 to 31 March 2016, and downloaded by the ESA portal at the address ftp://swarm-diss.eo.esa.int upon registration.

During this time interval Swarm A flew in polar orbit at an average altitude of about $460 \mathrm{~km}$, with an orbit in- 
clination of $87.4^{\circ}$. The satellite provided us with the following information: $i$ ) the UTC time, $i$ ) the position of the satellite in Earth-centered geographic coordinates GLat-GLong and $\mathrm{z}$ (altitude), iii) the in situ high resolution $(2 \mathrm{~Hz}$ ) electron density measured by the Langmuir probes of the Electric Field Instrument [EFI, Knudsen et al., 2017] in the North-East-Centre (NEC) frame of reference. Electron density data have been carefully examined and those suspicious have been excluded considering the information coming from the quality flag. It is due remembering that Swarm plasma data are still undergoing thorough validation by the experts of the mission.

Being interested in the variation of the electron density at mid and high latitudes in both the hemispheres and according to different geomagnetic activity levels, we used the Auroral Electrojet [AE, Davis and Sugiura, 1966] index to identify different geomagnetic conditions. This index, available with 1 minute cadence and based on the magnetic measurements from 12 observatories in the Northern Hemisphere, can be used to monitor the level of geomagnetic disturbance resulting from the eastward and westward auroral electrojets, being a measure of the total currents in the auroral zone. Using this index, we selected three different geomagnetic periods: quiet ( $\mathrm{AE}<50 \mathrm{nT}$ ), disturbed (50 nT $<\mathrm{AE}<250 \mathrm{nT}$ ) and highly disturbed $(\mathrm{AE}>250 \mathrm{nT}$ ). These thresholds were determined analysing the cumulative distribution of the $\mathrm{AE}$ index values in the selected time period. The value of $\mathrm{AE}$ $=50 \mathrm{nT}$, which identifies a geomagnetic quiet period, corresponds to the value of the $25^{\text {th }}$ percentile, while the value of $\mathrm{AE}=250 \mathrm{nT}$ corresponds to the $75^{\text {th }}$ percentile and identifies a geomagnetic highly disturbed period. Figure 1 reports the cumulative distribution of AE values in the time interval 1/04/2014 - 31/03/2016 and the thresholds used to pick out the three different geomagnetic conditions.

As the geomagnetic field is the main element of all the physical processes occurring in the magnetosphereionosphere system, and as the purpose of this work is to study the variations of the electron density and the scaling properties of its fluctuations at Swarm A altitudes under different geomagnetic activity levels, it comes natural to move from NEC to a magnetic frame of reference. The non-orthogonal Quasi-Dipole (QD) system is one of the most used in literature [Richmond 1995; Emmert et al. 2010; Laundal and Richmond, 2017]. It is based on the definition of the geomagnetic apex, i.e. the point on a field line at the maximum height above the reference ellipsoid (semimajor equatorial axis, $R_{E}$, of $6378.137 \mathrm{~km}$, ellipticity of 1/298.51) modelling the Earth. In this system the longitude, $\phi_{c d}$, is the center dipole longitude of

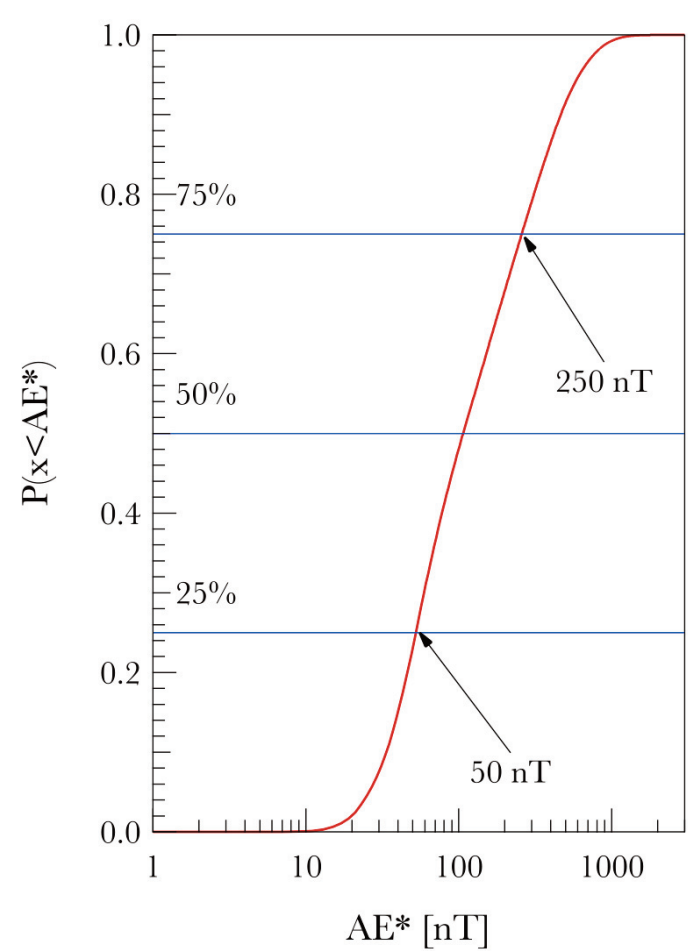

FIGURE 1. Cumulative distribution of the $\mathrm{AE}$ index in the time interval 1/04/2014 -31/03/2016. The value $\mathrm{AE}=50 \mathrm{nT}$ corresponds to the $25^{\text {th }}$ percentile, while the value of $\mathrm{AE}=250 \mathrm{nT}$ corresponds to the $75^{\text {th }}$ percentile.

the apex location, while the QD latitude is defined as

$$
\lambda_{Q D}= \pm \cos ^{-1}\left(\sqrt{\frac{R_{E}+h}{R_{E}+h_{a}}}\right)
$$

where $h$ is the distance from the geoid surface, $h_{a}$ is the apex height above the reference ellipsoid, and the positive (negative) sign holds in the Northern (Southern) Hemisphere. QD coordinates show two undoubted advantages when compared to other reference systems. Firstly, with respect to the orthogonal systems they are well defined and able to capture any feature at all the latitudes. Secondly, with respect to the other non-orthogonal systems they are suitable to locate phenomena with a specific height distribution (due to their dependence on $h$ ), like those at the Swarm A altitudes. The transformation from NEC to QD coordinates was performed according to the following steps: 1 ) we made the transformation from geocentric latitude, longitude and satellite altitude to geodetic latitude, longitude and altitude, respectively, following the algorithm described in Keeler and Nievergelt [1998]; 2) we followed the algorithm described in Emmert et al. [2010] to move from the geodetic to the QD system of coordinates. A robust and documented version of this algorithm is available in a 
package for Python language at the address https://apexpy.readthedocs.io/en/latest/index.html.

In order to consider the position of the Sun in this grid of coordinates we used, instead of the UTC time provided by Swarm A, the Magnetic Local Time (MLT). At each satellite location MLT can be defined as the difference in QD (or geodetic) longitude between the subsolar point and the location under consideration. In formula

$$
M L T=U T C+\frac{\phi_{c d}}{15}
$$

where of course $\phi_{c d}$ is expressed in degrees.

To analyse the features of the electron density and its
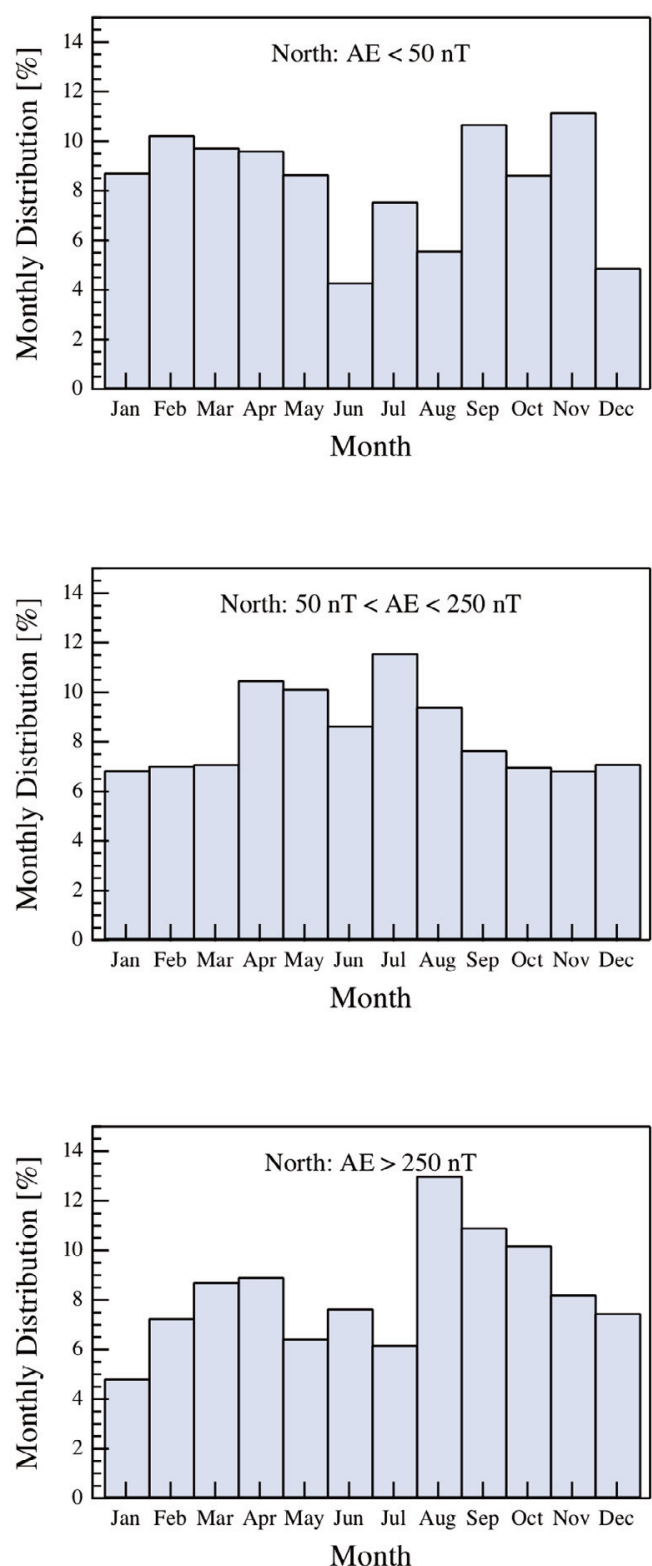

fluctuations at mid and high latitudes under different geomagnetic activity levels, we restricted to the range $\left|\lambda_{Q D}\right| \geq 50^{\circ}$ and represented our results in polar view maps in MLT vs $\lambda_{Q D}$. According to all the selection criteria, the three disturbance levels were sampled, respectively, with a number of values equal to $2.4 \cdot 10^{6}$ (in quiet conditions), $5.5 \cdot 10^{6}$ (in disturbed conditions), $2.9 \cdot 10^{6}$ (in highly disturbed conditions) in the Southern Hemisphere, and equal to $2.4 \cdot 10^{6}$ (in quiet conditions), $5.5 \cdot 10^{6}$ (in disturbed conditions), $2.9 \cdot 10^{6}$ (in highly disturbed conditions) in the Northern Hemisphere. Figure 2 reports the monthly distribution of our data set according to the three geomagnetic activity levels in both the Hemispheres.
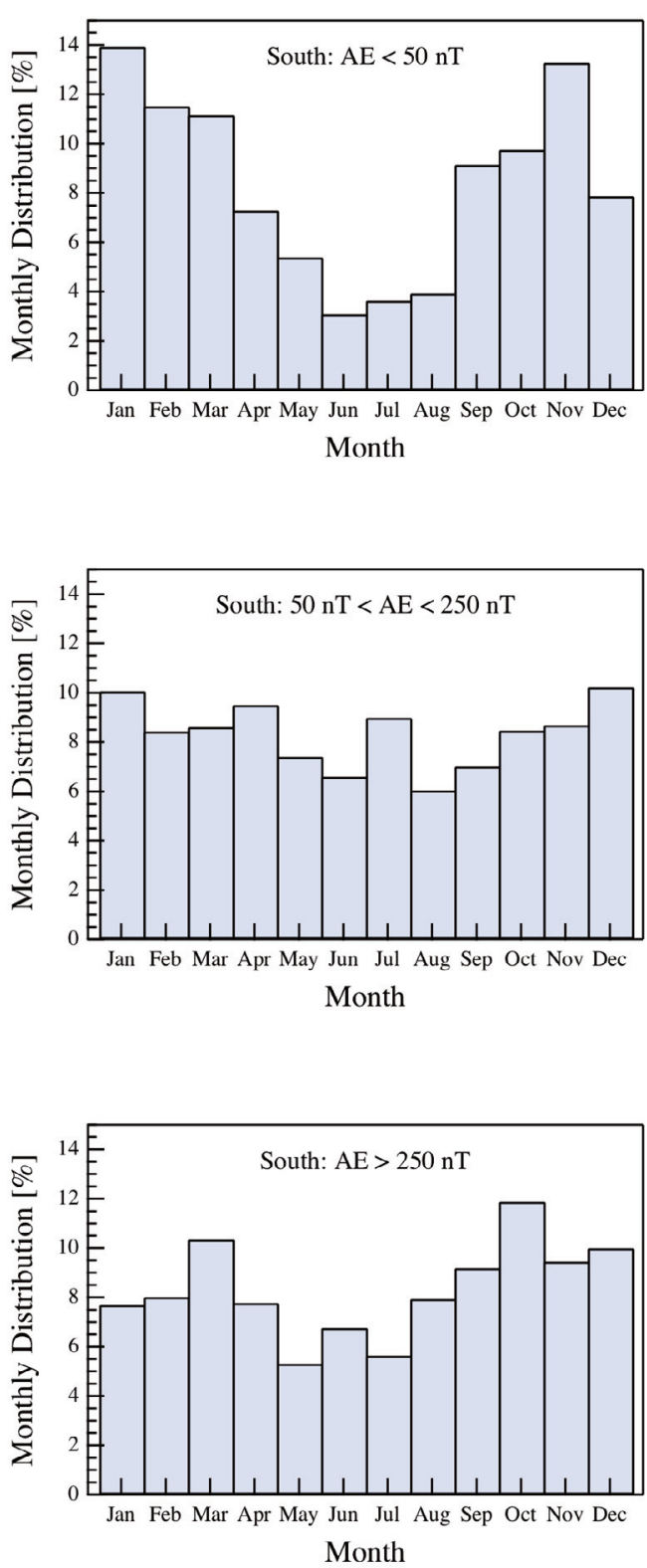

FIGURE 2. Monthly distribution of sampled data according the three geomagnetic activity levels: AE < $50 \mathrm{nT}$ (top panel), $50 \mathrm{nT}<$ $\mathrm{AE}<250 \mathrm{nT}$ (middle panel), and AE > $250 \mathrm{nT}$ (bottom panel) for both the Northern (left column) and Southern (right column) Hemispheres. 


\section{METHOD OF ANALYSIS}

When the fluctuations associated with physical quantities are large enough and the transfer of energy occurs via a nonlinear cascade mechanism that involves many spatial and temporal scales, the system is in a turbulent state. Indeed, according to Falkovich [2008] the word turbulence indicates "a state of a physical system with many interacting degrees of freedom deviated far from equilibrium" that is "irregular both in time and in space and is accompanied by dissipation".

One of the possible indication of the existence of the turbulent regime in the ionosphere is the power-law behaviour of the statistical or spectral properties of physical quantities [see, e.g., Kintner Jr., 1976; Sugiura et al., 1982; Heppner et al., 1993; Abel and Freeman, 2002; Abel et al., 2002; Pulkkinen et al., 2006; Golovchanskaya et al., 2006; Abel et al., 2007; Kozelov et al., 2008; Golovchanskaya and Kozelov, 2010; Cousins and Shepherd, 2010; De Michelis et al., 2015a, 2015b, 2016]. For this reason, in this work, we focus on the analysis of the scaling properties of the ionospheric electron density to characterize its possible turbulent state, which may play a key role in the generation and in the dynamic of ionospheric inhomogeneities.

Among the analysis techniques known by far, those based on the structure functions [Frisch, 1995] have been extensively used to capture the turbulent state of the ionospheric medium, and in general to study all those phenomena where turbulence is thought to play a fundamental role in the transfer of energy across a wide range of spatiotemporal scales.

Given a time series, like in our case the electron density $N_{e}(t)$ at the generic scale $t$ (time, in this case), the $q$-th order structure function $S_{q}(\tau)$ associated with the perturbation scale $\tau$ is defined as

$$
S_{q}(\tau)=\left\langle\left|N_{e}(\mathrm{t}+\tau)-N_{e}(\mathrm{t})\right|^{q}\right\rangle_{T}
$$

where the ensemble average $<\ldots>$ runs over all the pairs separated by $\tau$, which is the current scale of interest, over the time interval $T$. For a stationary and scale-invariant electron density series, the structure function behaves like a power law of the perturbation $\tau$ with scaling exponent $\xi(q)$, namely

$$
S_{q}(\tau) \propto \tau^{\xi(q)}
$$

In particular, the first- order structure function $S_{1}(\tau)$ can be rewritten as

$$
S_{1}(\tau) \propto \tau^{H}
$$

being $H \equiv \xi(1)$ the Hurst exponent [Hurst, 1956]. This quantity, which can take the values in the range [0, 1], is a direct measure of the long-term memory of the series $N_{e}(t)$, and can be used to get insights on its dynamical behaviour. In other words, $H$ can be considered as a benchmark for persistency, anti-persistency or randomness in $N_{e}(t)$ analysis: when $H \in(0,0.5)$ the series follows an antipersistent behaviour, meaning that an increase (decrease) of $N_{e}(t)$ will be statistically followed by a decrease (increase) in order to bring $N_{e}(t)$ closer to the stationary value and to induce stability in the dynamical system. Conversely, when $H \in(0,5.1)$ the series follows a persistent behaviour, meaning that an increase (decrease) of $N_{e}(t)$ will be statistically followed, again, by an increase (decrease), giving rise to a trending behaviour of $N_{e}(t)$, which clusters the fluctuations along a direction. $0 n$ the extremes, $H=0.5$ corresponds to randomness, i.e. a situation of complete loss of correlation (or anticorrelation), while $H=1$ corresponds to a linear predictable trend of $N_{e}(t)$.

In recent literature, the Hurst exponent has been successfully used to characterise the correlations and the persistent features inherent to magnetic field fluctuations observed from the ground [Balasis et al., 2006, 2008, 2009; De Michelis et al., 2015a] and via in situ ionospheric measurements performed by the Swarm mission [De Michelis et al., 2015b; De Michelis et al., 2016; De Michelis et al., 2017]. In this work, we applied the Hurst exponent technique to the electron density fluctuations measured by Swarm in the ionospheric Flayer by performing the same approach of the detrended structure function analysis (DSFA) used by De Michelis et al. [2015a] and consisting in the following steps. We set the range of fluctuation scales $\tau$ between $1 \mathrm{~s}\left(\tau_{\text {min }}\right)$ and $40 \mathrm{~s}\left(\tau_{\max }\right)$. We performed the analysis of this data set in the temporal domain with the implicit use of the Taylor's frozen hypothesis [Taylor, 1938] to convert the temporal data to spatial ones. Formally, Taylor's hypothesis assumes that the advection velocity of turbulence is much greater than the velocity scale of turbulence itself. To quote the original statement in Taylor [1938]: "If the velocity of the air stream which carries the eddies is very much greater than the turbulent velocity, one may assume that the sequence of changes in $u$ at a fixed point are simply due to the passage of an unchanging pattern of turbulent motion over the point”. By invoking this assumption, time is replaced with a distance scaled by velocity. Thus, taking into consideration that the orbital velocity of the satellite is $\sim 7.6 \mathrm{~km} / \mathrm{s}$, the range of fluctuation scales $\tau$ between 1 $\mathrm{s}$ and $40 \mathrm{~s}$ corresponds to the range of spatial fluctuations between $\sim 8 \mathrm{~km}$ and $\sim 300 \mathrm{~km}$. The time series $N_{e}(t)$ 
was then detrended by subtracting to the signal a seventh-order polynomial. This allowed us to remove the low frequency trends and to compute the time-varying Hurst exponents in steps of $T$, where $T$ is equal to 400 $\mathrm{s}$ that means at least 10 times larger than the maximum scale $\tau$ that we want to investigate. For the sake of simplicity we will refer to the detrended electron density series as, again, $N_{e}(t)$. Once set the triad $\tau_{\min }, \tau_{\max }$ and $T$ we computed in each moving time window the firstorder structure function and fitted to a power law in order to retrieve $H$ from equations 3 and 5, namely

$$
S_{1}(\tau)=\left\langle\left|N_{e}(\mathrm{t}+\tau)-N_{e}(\mathrm{t})\right|\right\rangle_{T} \propto \tau^{H}
$$

For a self-affine fractal signal (such as a fractional Brownian motion) characterized by an Hurst exponent, $\mathrm{H}$, the power spectral density $S(f)$ is expected to scale, again, according to a power law $S(f) \propto f^{-\beta}$ where the spectral index $\beta$ is related to the Hurst exponent via the relation

$$
\beta \simeq 2 \mathrm{H}+1
$$

showing that different values of $H$ correspond to different values of the spectral index $\beta$ [Bunde \&t Havlin, 1995]. Thus, in our analysis the knowledge of the Hurst exponent of electron density fructuations may also provide information on the energy spectral content of fluctuations and consequently on the dynamic properties of the ionosphere [Balasis et al., 2006, 2009, 2013]. However, we remark that previous Eq. (7) is valid under the assumption that there are no corrections due to intermittency otherwise the expected value of the spectral index is $\beta<2 H+1$. As $H$ lies in the range [0,1], the spectral index $\beta$ spans the range [1,3]. In particular, in the case of an antipersistent behaviour of fluctuations $(H>0.5) \beta<2$ holds; while for persistent fluctuations $(H>0.5) 2<\beta<3$ holds.

\section{RESULTS AND DISCUSSION}

In this section we are going to present the results of the analysis of the electron density and scaling features in term of polar maps. These maps have been obtained using an averaging procedure based on a distance-kernel method [see for details De Michelis et al., 2015b].

Figure 3 reports the large-scale spatial distribution of the electron density $\left(N_{e}\right)$ in both the Northern and Southern Hemispheres $\left(\left|\lambda_{O D}\right| \geq 50^{\circ}\right)$ on polar view maps at Swarm A altitude, for three different levels of geomagnetic activity (quiet: $\mathrm{AE}<50 \mathrm{nT}$, disturbed: $50 \mathrm{nT}<$
$\mathrm{AE}<250 \mathrm{nT}$ and highly disturbed: $\mathrm{AE}>250 \mathrm{nT})$. In the Northern Hemisphere the electron density takes values roughly in the range between $0.6 \cdot 10^{5} \mathrm{~cm}^{-3}$ and $2 \cdot 10^{5}$ $\mathrm{cm}^{-3}$. The maximum electron density is observed at noon between $60^{\circ}$ and $70^{\circ}$ of magnetic latitude, while the minimum lies in the night sector between $50^{\circ}$ and $60^{\circ}$ of magnetic latitude. Several typical features of the polar ionosphere, obtained in the past using several in situ and remote techniques, can be recognised looking at these maps: 1) The electron density in the dayside is almost twice that in the nightside due to the strong ultra-violet (UV) ionization of the Earth upper atmosphere, regardless of the geomagnetic activity level; 2) The tongue of ionization (TOI), which extends from noon across the polar cap to the nightside [Knudsen, 1974; Foster et al., 2005], is well visible when the geomagnetic disturbance level increases and acts to disrupt the steep density gradient between the morning and the night sectors; 3) The increase of electron density, known as "dusk effect" or "Storm time Enhanced Density" [SED, Foster, 1993], is recognizable around dusk during disturbed periods; and 4) A depletion in the electron density, which identifies the Main Ionospheric Trough (MIT) can be observed in the sub-auroral ionospheric region, primarily in the night side.

In the Southern Hemisphere the spatial distribution of the electron density is different from that obtained in the Northern Hemisphere. The differences are evident mainly in the quiet and moderately disturbed periods for almost all MLTs. A general comparison between the two Hemispheres shows electron density values that are generally higher in the Southern Hemisphere than in the Northern one. The difference in the electron density values between the two Hemispheres is visible especially at low latitudes in the night sector. These differences could be a consequence of both seasonal effects and the different magnitude and distribution of the geomagnetic field. It is known indeed that in the Southern Hemisphere, due to both the offset between the geographic and magnetic poles and to the Sun-Earth distance, the exposure to UV radiation is greater than in the Northern Hemisphere. This highest exposure to UV radiation explains our general results, governing the UV radiation the production and loss of electrons. However, the uneven distribution of data in the local seasons in the two hemispheres can be responsible for a seasonal effect probably present in the Southern Hemisphere, where the spring and summer periods are more sampled, especially under quiet conditions (Figure 2).

By looking at the change of the electron density distribution with the geomagnetic activity levels we notice that the most prominent electron density enhancements 
occur in the polar cap. These enhancements can be associated with the polar cap patches, which are defined as regions with F-layer plasma densities 2-10 times larger than the background density that are thought to originate some distance away and then be drifted by the polar convection. The electron density enhancement due to the precipitation of low-energy electrons $(E<300 \mathrm{eV})$ and the change of circulation triggered by an increase of the solar wind flow or by a sudden change of the interplanetary magnetic field (IMF) orientation [see, e.g., Anderson et al., 1998], are among the possible origins of these spatial gradients, which during geomagnetic active periods are transported across the pole via global convection providing the ionization along the path toward the midnight and the formation of the TOI. Once reached the Harang discontinuity, plasma moves eastward and westward again toward the noon. This picture is consistent with the results shown Figure 3, where it is shown the density increase with the geomagnetic activity at very high latitudes.
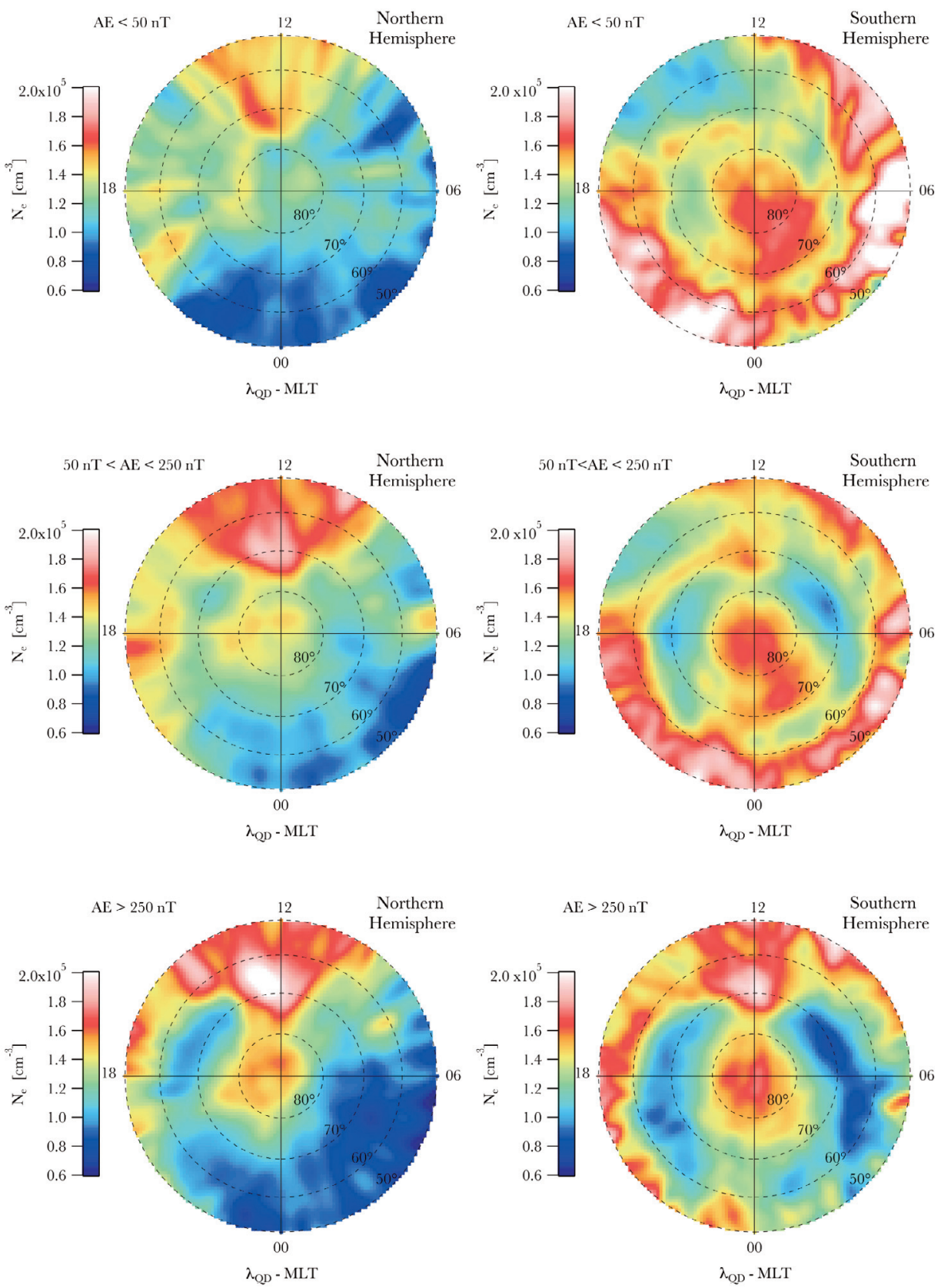

FIGURE 3. On the left: polar view of spatial distribution of the mean electron density (Ne), in the Northern Hemisphere, in $\lambda_{\text {QD }}$ coordinates $\left(50^{\circ} \mathrm{N}-90^{\circ} \mathrm{N}\right)$ and MLT during quiet $(\mathrm{AE}<50 \mathrm{nT})$, disturbed $(50 \mathrm{nT}<\mathrm{AE}<250 \mathrm{nT})$ and highly disturbed geomagnetic conditions (AE>250 nT). The four dashed circles mark the latitudes of $50^{\circ}, 60^{\circ}, 70^{\circ}$ and $80^{\circ}$. On the right: same view as described above, but concerning the Southern Hemisphere. 
In order to obtain a measure of the variability field of the electron density we performed a local variance analysis. In particular, we retrieved the standard deviation, $\sigma_{\mathrm{Ne}}$, maps of the electron density in the mid-and high- latitude regions of both the Northern and Southern Hemispheres. This provided a statistical indication of the local spread of electron density around the mean value, or in other words of the local amplitude of electron density fluctuations. Furthermore, variance, $\sigma_{\mathrm{Ne}}^{2}$, is related to the spectral energy content of density fluctuations, and thus may give clues of the dynamic state of a system. We underline that in some known turbulent systems the fluctuations amplitude is typically of the same order of magnitude of the mean value. Figure 4 reports the spatial distribution of the ratio between the standard deviation of electron density $\left(\sigma_{\mathrm{Ne}}\right)$ and its mean value $\left(N_{\mathrm{e}}\right)$ according to the three selected geomagnetic activity levels in both the hemispheres. The values are generally high, suggesting the existence of a turbulent regime in each of the analysed ionospheric regions. Such a complex behaviour is probably due to the onset of the instabilities rising as a consequence of the electron density gradients in the high-latitude ionosphere.

Among the suggested mechanisms operating to generate these instabilities we mention the $\mathrm{E} \times \mathrm{B}$ current convective instability at intermediate scales (between $100 \mathrm{~m}$ and $10 \mathrm{~km}$ ), which can be driven by field aligned currents and electric fields, and the occurrence of drift waves at smaller scales [see, e.g., Kelley et al., 1982]. It is interesting to notice that the values of $\sigma_{\mathrm{Ne}} / N_{\mathrm{e}}$ are generally higher in the Southern Hemisphere than in the Northern one, especially at lower latitudes in the nightside. Lower latitude regions are characterised by the presence of steep density gradients and could drive processes of plasma instability on the base of the turbulent behaviour. In fact, it is reasonable to suppose that the increase of turbulence in these regions is the result of various inhomogeneities and nonstationarities in the magnetospheric plasma that are projected to these ionospheric regions through the magnetic field lines. The ultimate state in both the hemispheres at high latitudes is a diffusion of higher density fluctuation amplitudes indicating that the system is more "chaotic" in response to the increased geomagnetic activity. We also note a strong enhanced variability field of the electron density in the dawn sector with respect to the dusk one, which also leads to an increase of the asymmetry between dusk and dawn sectors. This enhancement and the following asymmetry is probably the result of the strong electron density gradients arising in correspondence to both dawn and dusk. These gradients, at MLT
06:00 are generally steeper at lower latitudes than at the higher ones, as toward the pole the length of the daytime (nightime) is longer and longer, thus there is not an abrupt change of the ionization conditions in the F-layer of the ionosphere within 24 hours. As electron density gradients generate instabilities leading to turbulence, it is reasonable to expect a higher degree of turbulence in correspondence to MLT 06:00. Moreover, as the fluctuations amplitude represents a proxy of the degree of turbulence, this argument can explain the strong enhancement in the standard deviation maps of the electron density shown in Figure 4.

Figure 5 reports the large-scale spatial distribution of the Hurst exponent, $H$, associated with the electron density series in both the Northern and the Southern $\left(\left|\lambda_{Q D}\right| \geq 50^{\circ}\right)$ Hemispheres on polar view maps for three different levels of geomagnetic activity (quiet: $\mathrm{AE}<50$ nT, disturbed: $50 \mathrm{nT}<\mathrm{AE}<250 \mathrm{nT}$ and highly disturbed: AE>250 nT). As we can see, the values of $H$ basically range between 0.2 and 0.5 during quiet and disturbed geomagnetic activity, while the maximum value of $H$ is $>0.5$ during highly disturbed periods and around dusk (white regions in the bottom panels of Figure 5). Moreover, the spatial structure of $H$ is the same in both the hemispheres, with the $H$ values slightly higher in the Southern Hemisphere than in the Northern one. This indicates that the electron density fluctuations have an antipersistent character in both the hemispheres at high latitudes when $\mathrm{AE}<250$ nT. This means that in the analysed regions the electron density responds to perturbations in order to damp the large-scale fluctuations and bring the electron density back to the stationary value, thus generating essentially small-scale structures. This feature depicts an important property inherent to the dynamical behaviour of the ionosphere. In quiet periods, the minimum $H$ (maximum antipersistence) is observed in the dayside around noon, and in correspondence to the polar cap. Probably in these regions the solar ionisation and the particle precipitation, respectively, have a stabilizing effect on the density fluctuations, generating very small-scale structures. We also find that the antipersistent character endures even under disturbance conditions, but tends to be less and less pronounced with the increasing of the geomagnetic activity level. Finally, during highly disturbed periods a slightly persistence character is observed around dusk in both the hemispheres. This suggests that the internal stabilizing processes driving the antipersistence are less and less effective in bringing back the system to stationary conditions in presence of stronger and stronger externally-induced disturbances that tend to more and more increase the electron density fluctuations. In pre- 
vious studies, the persistent character of the horizontal geomagnetic field fluctuations was associated with the onset of global convection patterns at high latitudes in different IMF orientations [De Michelis et al., 2017]. In the light of this, the reduced antipersistence during more disturbed periods could be associated with the enhancement of the convection patterns driven by the electric field forcing the open geomagnetic field lines. However, this correspondence is far away to be obvious and will be investigated in a future work. The only re-

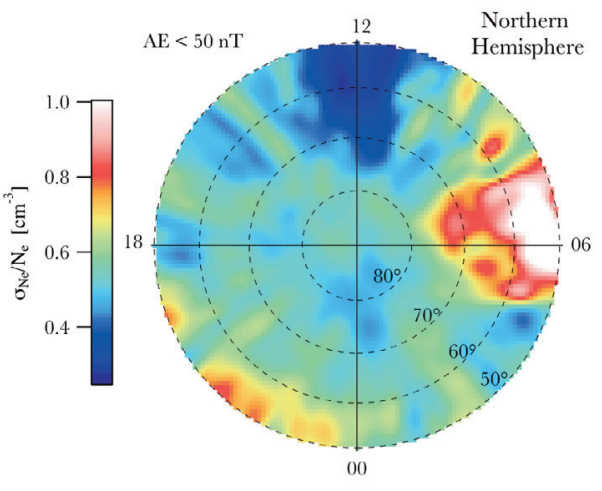

$\lambda_{\text {QD }}-$ MLT
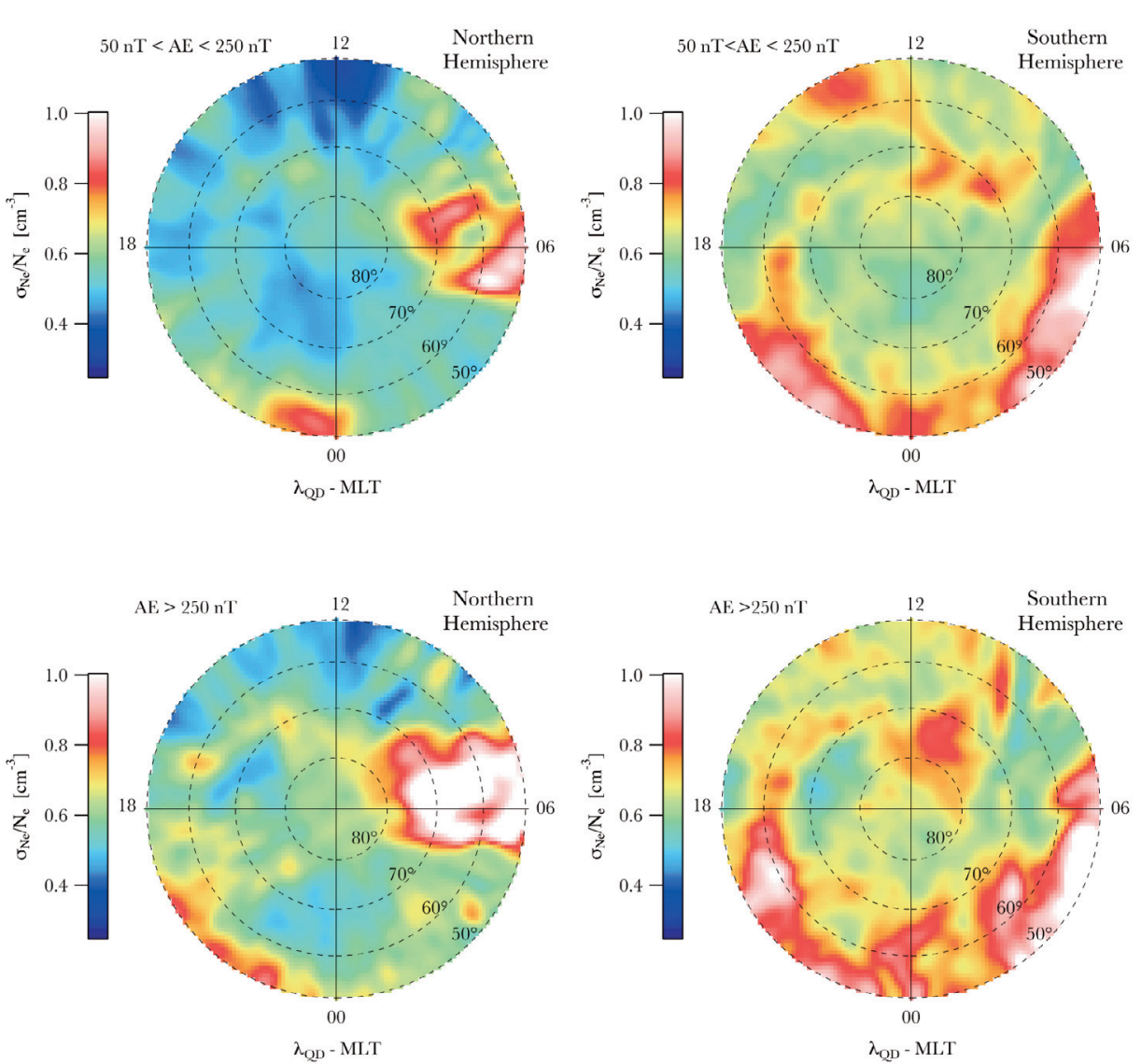

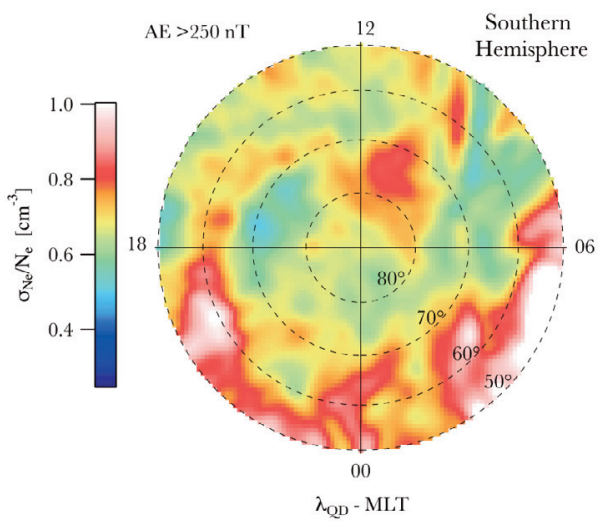

gion where the density fluctuations assume a character manifestly persistent with the increase of the geomagnetic activity is nearby dusk between magnetic latitudes $50^{\circ}$ and $60^{\circ}$. This feature might be a manifestation of the dusk effect and of the marked increase of the density fluctuations with the geomagnetic activity.

From the spectral point of view, the values of $H<0.5$ retrieved correspond to spectral indices $\beta<2$ characterising the energy spectral content of the fluctuations. To date, several studies have investigated the

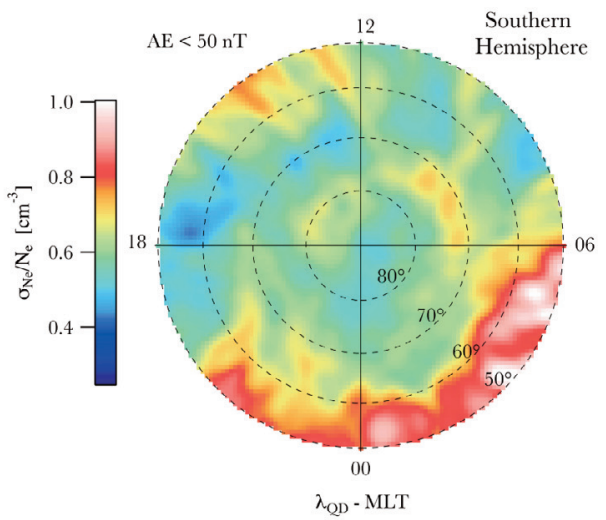

$\lambda_{\mathrm{QD}}-\mathrm{MLT}$

FIGURE 4. On the left: polar view of spatial distribution of the ratio between the standard deviation of the electron density and its mean value, $\sigma_{\mathrm{Ne}} / N_{e}$, in the Northern Hemisphere, in $\lambda_{\mathrm{OD}}$ coordinates $\left(50^{\circ} \mathrm{N}-90^{\circ} \mathrm{N}\right)$ and MLT during quiet (AE $\left.<50 \mathrm{nT}\right)$, disturbed ( $50 \mathrm{nT}<\mathrm{AE}<250 \mathrm{nT})$ and high disturbed $(\mathrm{AE}>250 \mathrm{nT})$ geomagnetic conditions. The four dashed circles mark the latitudes of $50^{\circ}, 60^{\circ}, 70^{\circ}$ and $80^{\circ}$. On the right: same view as described above, but concerning the Southern Hemisphere. 
electron density spectra in the ionospheric F-layer, founding that they can be fairly described by power laws with typical spectral index of 1.9 [see, e.g., Dyson et al., 1974] with other reported values ranging from 1.5 to 2.5 [see, e.g., Villain et al., 1986]. These spectral indices obtained using in situ satellite measurements of electron density, are relative to spatial scales from $\sim 40$ $\mathrm{m}$ to $\sim 100 \mathrm{~km}$. Similar values have been obtained from in situ rocket measurements, while spectral indices retrieved from scintillation measurements were found to be slightly lower: $\sim 1.4$ in the auroral zones and $\sim 1.3$ in the polar cap [(Basu et al., 1985]. However, there are studies where the electron density spectra are better fitted by two power laws with different spectral indices, in which the high frequency component is steeper than the lower one, whose spectral index is consistent with those found in the case of a single power law, with the knee occurring at a scale of $\sim 300 \mathrm{~m}$ [Villain et al., 1986]. Generally speaking, the spatial scales involved in deriving the electron density spectra are separated in the
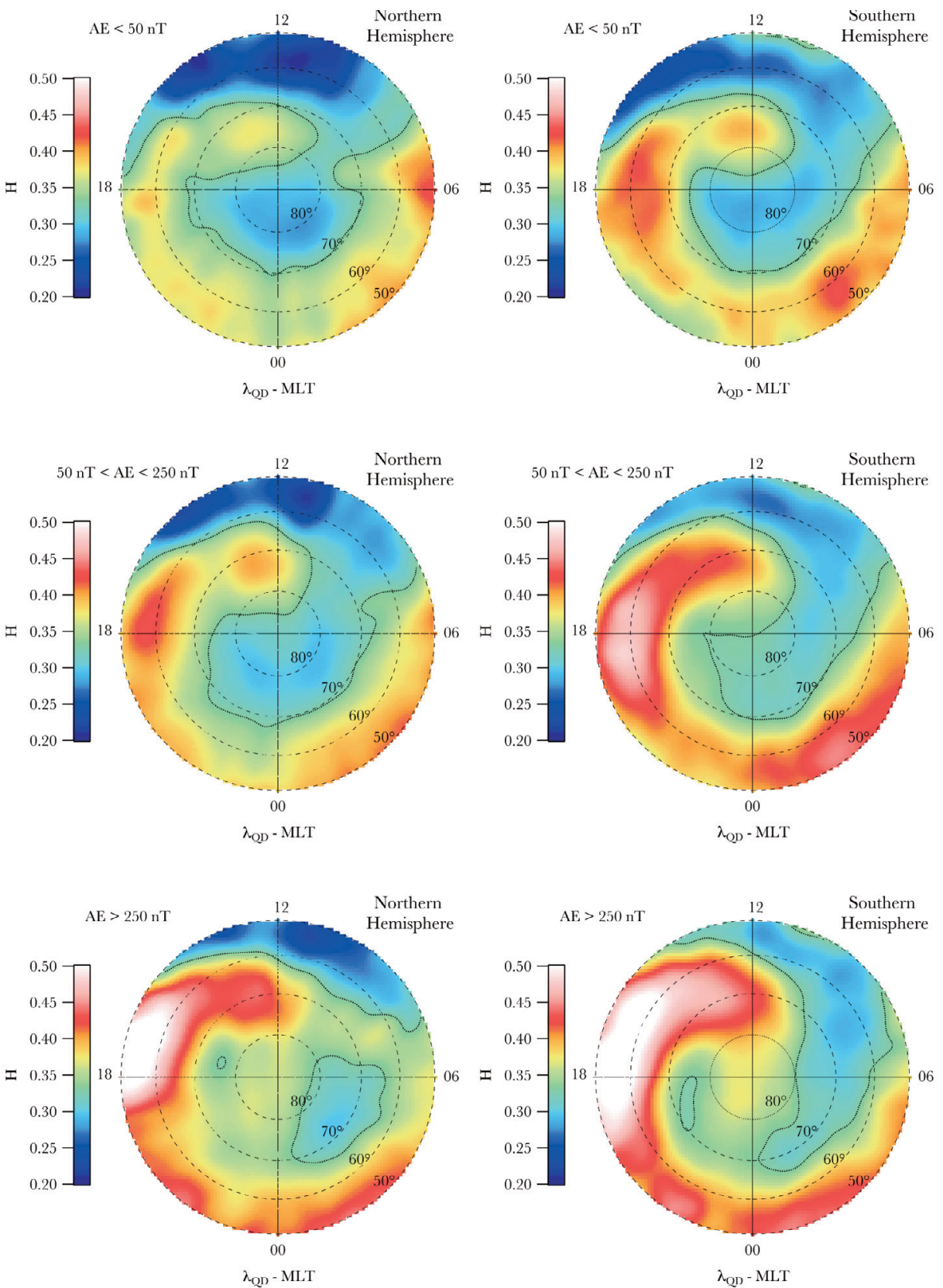

FIGURE 5. On the left: a polar view of spatial distribution of the local Hurst exponent values $(\mathrm{H})$, in the Northern Hemisphere, in $\lambda_{\mathrm{OD}}$ coordinates $\left(50^{\circ} \mathrm{N}-90^{\circ} \mathrm{N}\right)$ and MLT during quiet (AE $\left.<50 \mathrm{nT}\right)$, disturbed (50 nT $<\mathrm{AE}<250 \mathrm{nT}$ ) and high disturbed (AE $>250$ nT) geomagnetic conditions. The dotted line corresponds to $\mathrm{H}=0.33$ and the four dashed circles mark the latitudes of $50^{\circ}$, $60^{\circ}, 70^{\circ}$ and $80^{\circ}$. On the right: same view as described above, but concerning the Southern Hemisphere. 
following regimes [Kelley, 1985]: long $(1 / K>20 \mathrm{~km})$, intermediate $(100 \mathrm{~m}<1 / K<20 \mathrm{~km})$, transitional $(10 \mathrm{~m}$ $<1 / K<10 \mathrm{~m})$, and short $(0.1 \mathrm{~m}<1 / K<10 \mathrm{~m})$. In this study we are limited to accessible scales lying in the range $8-300 \mathrm{~km}$, crossing the so-called long and intermediate regimes. However, the common feature of all these results is the finding of power law spectra with indices between 1 and 3. As it was clear since the first studies on this topic, the presence of power laws indicates self-similarity, according to which the same physical processes operate at all the electron density scales available, and there is no evidence of preferred scales. On the contrary, the presence of more spectral indices indicates an intermittent character due to the presence of different energy transfer and dissipation mechanisms within the system, and to the local properties of the medium. By looking at the maps in Figure 4, we argue that the values of the spectral index retrieved in our study may be associated with different turbulent regimes like, e.g., the shear flow turbulence with an inverse energy cascade (i.e., the transfer of energy occurs from the small scales to the large ones); the turbulence driven by the $\mathrm{E} \mathbf{x}$ B current convective instability mentioned above; or the turbulence driven by strong electron density gradient drifts [Kintner Jr., 1976]. In particular, the value $H=0.33$ of the iso-contours in the panels of Figure 5 corresponds to a spectral index $\beta=1.33$ under the hypothesis of no-intermittency corrections. This spectral index well separates the regions whose spectra are only slightly changed by the geomagnetic activity. These sorts of islands are characterised by a little change in the dynamical properties with respect to the other regions. Moreover, the value $H=0.33$, which corresponds to the first-order structure function $S_{1}$, is the value expected by the Kolmogorov theory K41 in fluid turbulent media in absence of intermittency, which implies a correction to a typical value of about $H \simeq 0.73$ [Ruiz-Chavarria et al., 1996]. This means that the dotted contour in Figure 5 well separates different turbulent regimes, being the classical K41 a smooth transition boundary between them. However, we remark that a better way to describe the dynamical evolution of the electron density in the ionosphere would be that of considering this quantity as a passive scalar that evolves according to the following equation [see, e.g., Celani et al., 2002]

$$
\frac{\partial N_{e}}{\partial T}+(\vec{v} \cdot \nabla) N_{e}=\chi \nabla^{2} N_{e}+f
$$

where $\vec{v}$ is the plasma velocity field, $\chi$ the diffusivity coefficient and $f$ a source term. In such a theoretical framework the emergence of turbulence would cause a rapid growth of intermittent fluctuations, so that intermittency corrections to the Hurst exponent have to be included to provide a more reliable scenario. This study is demanded to a future work.

The only region that shows a persistent character in both the Hemispheres with the increase of geomagnetic activity (in white in the bottom panels of Figure 5) is associated with a spectral index $\beta>2$, that may be the result of a strong plasma shear flow regime operating on the system [Sridhar and Goldreich, 1995].

\section{SUMMARY AND CONCLUSIONS}

The purpose of the current study was to perform a nonlinear analysis capable of providing information on the dynamical state of the mid and high-latitude electron density and it fluctuations. For this reason, electron density measurements recorded onboard the Swarm A satellite during a period of two years were considered and analysed according to three different geomagnetic activity levels: low (AE $<50 \mathrm{nT}$ ), moderate (50 nT $<\mathrm{AE}$ $<250 \mathrm{nT}$ ), and high ( $\mathrm{AE}>250 \mathrm{nT})$. The main results may be briefly resumed in the following points:

1) The density maps show all the well known main features of the mid- and high- latitude ionosphere, like the sunlit enhancement at noon by solar UV ionization of the Earth upper atmosphere; the presence of a TOI crossing the polar cap and disrupting the expected steep density gradients between the morning and night sectors under intense geomagnetic activity levels; the presence of the depleted main trough at the equatorward boundary of the auroral regions signing the presence of the plasmapause; the presence of a density enhancement nearby the dusk.

2) The standard deviation of the electron density over its mean values gives an estimation of the variability field of this quantity or equivalently of the amplitude of the electron density fluctuations. This is important because high values of this ratio are expected in regions where turbulence plays a fundamental role. Such a complex dynamical state is triggered by the instabilities arising as a consequence of electron density gradients occurring in the high-latitude ionosphere. In quiet conditions the ratio takes values up to $\sim 0.4$ around noon, and up to $\sim 0.8$ in the night sector at middle latitudes. A peak is observed nearby dawn.

3) The spatial distributions of the local Hurst exponent values, $H$, show their change with the geomagnetic conditions. The Hurst exponent maps, 
based on the computation of the first-order structure functions, point out the antipersistent character of the electron density fluctuations over the whole Northern and Southern Hemispheres at mid and high latitudes. This means that as a consequence of density fluctuations the system rapidly responds in order to bring back the density to its stationary value. The minimum $H$ occurs in the dayside and in correspondence to the polar cap, probably where the solar ionization and the particle precipitation have a stabilizing effect on the fluctuations. We also find that the antipersistent character tends to be less and less pronounced during increased geomagnetic activity. This means that the internal stabilizing processes are less and less effective in bringing back the system to stationary conditions in presence of externally induced disturbances that tend to more and more increase the electron density fluctuations.

Our findings are consistent with a turbulent interpretation of the ionospheric dynamics, and with the presence of different turbulent regimes in different locations and different geomagnetic activity conditions. We believe this work may stimulate a constructive debate in the community, playing the turbulence a fundamental role in the ionospheric dynamics in response to its coupling with the magnetosphere and ultimately with the solar wind. Furthermore, it will contribute to better understand the actual ionosphere state during both quiet and active periods, and hopefully be able to perform reliable predictions of the occurrence of fluctuations that may represent a Space Weather hazard.

ACKNOWLEDGEMENTS. The results presented in this paper rely on data collected by one of the three satellites of the Swarm constellation. We thank the European Space Agency that supports the Swarm mission. Swarm data can be accessed online at http://earth.esa.int/swarm. The authors kindly acknowledge Papitashvili and J. King at the National Space Science Data Center of the Goddard Space Flight Center for the use permission of 1 min OMNI data and the NASA CDAWeb team for making these data available. G. Consolini and V. Quattociocchi thank the Italian Space Agency (ASI) for the financial funding under contract ASI "LIMADOU Scienza".

\section{REFERENCES}

Abel, G. A., and M. P. Freeman (2002). A statistical analysis of ionospheric velocity and magnetic field power spectra at the time of pulsed ionospheric flows. J. Geophys. Res., 1470 (A12), 1470.

Abel, G. A., M. P. Freeman, and G. Crisham (2002). Spatial structure of ionospheric convection velocities in regions of open and closed magnetic field topology. Geophys. Res. Lett., 33, L24103.

Abel, G. A., M. P. Freeman, G. Crisham, N. W. Watkins (2007). Investigating turbulent structure of ionospheric plasma velocity using the Halley SuperDARN radar. Nonlinear Processes in Geophysics, 14, 799-809.

Anderson, P. C., D. L. Chenette, D. L. McKenzie, J. M. Quinn, M. Grande, and M. Carter (1998), Energetic auroral electron distributions derived from global $\mathrm{X}$-ray measurements and comparison with in-situ particle measurements, Geophys. Res. Lett., 25, 4150-4108.

Balasis G., I. A. Daglis, P. Kapiris, M. Mandea, D. Vassiliadis, and K. Eftax-ias (2006), From pre-storm activity to magnetic storms: a transition described in terms of fractal dynamics, Ann. Geophys., 24, 3557-3567.

Balasis, G., I. A. Daglis, C. Papadimitriou, M. Kalimeri, A. Anastasiadis, and K. Eftaxias (2008), Dynamical complexity in Dst time series using non-extensive Tsallis entropy, Geophys. Res. Lett., 35, L14102, doi:10.1029/2008GL034743. 3

Balasis, G., I. A. Daglis, C. Papadimitriou, M. Kalimeri, A. Anastasiadis, and K. Eftaxias (2009), Investigating dynamical complexity in the magnetosphere using various entropy measures, J. Geophys. Res., 114, A00D06, doi:10.1029/2008JA014035.

Balasis, G., R. V. Donner, S. M. Potirakis, J. Runge, C. Papadimitriou, I. A. Daglis, K. Eftaxias, and J. Kurths (2013), Statistical mechanics and information-theoretic perspectives on complexity in the Earth system, Entropy, 15 (11), 4844-4888; doi:10.3390/e15114844.

Basu, S., E. MacKenzie, W. R. Coley, W. B. Hanson, and C. S. Lin (1984), F-Regon Electron Density Irregularity Spectra near Auroral Acceleration and Shear Regions, J. Geophys. Res. 89, 5554.

Basu, S., E. Mackenzie, and H. E. Whitney (1985), Morphology of phase and intensity scintillations in the auroral oval and polar cap, Radio Sci., 20,347.

Basu, S., E. Mackenzie, P. F. Fougere, W. R. Coley, N. C. Maynard, J. D. Winningham, M. Sugiura, W. B. Hanson, and W. R. Hoegy (1988), Simultaneous density and electric field fluctuation spectra associated with velocity shears in the auroral oval, J. Geophys. Res., 93(A1),115-136, doi:10.1029/JA093iA01p00115.

Bunde, A. and S. Havlin (1995), Fractals in Science, 
Springer-Verlag

Celani, A., M. Cencini, A. Mazzino, and M. Vergassola (2000), Active versus Passive Scalar Turbulence, Phys. Rev. Lett., 89, 234502.

Cousins, E. D. P., and S. G. Shepherd (2010). A dynamical model of high-latitude convection derived from SuperDARN plasma drift measurements. J. Geophys. Res., 115, A12329.

Davis, T. N., and M. Sugiura (1966). Auroral electrojet activity index $\mathrm{AE}$ and its universal time variations. J. Geophys. Res., 71, 785-801.

De Michelis, P., G. Consolini, and R. Tozzi (2015a), Magnetic field fluctuation features at Swarm's altitude: A fractal approach, Geophys. Res. Lett., 42, 31003105, doi:10.1002/2015GL063603.

De Michelis, P. and G. Consolini (2015b), On the local Hurst exponent of geomagnetic field fluctuations: Spatial distribution for different geomagnetic activity levels, J. Geophys. Res., 120, 2691-2701, doi:10.1002/2014JA020685.

De Michelis, P., G. Consolini, R. Tozzi, and M.F. Marcucci (2016), Observations of high-latitude geomagnetic field fluctuations during St. Patrick's Day storm: Swarm and SuperDARN measurements, Earth Planets Space, 68, 105, doi: 10.1186/s40623016-0476-3.

De Michelis, P., G. Consolini, R. Tozzi, and M.F. Marcucci (2017), Scaling features of high latitude geomagnetic field fluctuations at Swarm altitude: Impact of IMF orientation, J. Geophys. Res. Space Phys., 122, 10548-10562, doi:10.1002/2017JA024156.

Dyson, P. L., J. P. McClure, and W. B. Hanson (1974), In situ measurements of the spectral characteristic of F region ionospheric irregularities, J. Geophys. Res., 79, 1497.

Earle, G. D., M. C. Kelley, and G. Ganguli (1989), Large velocity shears and associated electrostatic waves and turbulence in the auroral F region, J. Geophys. Res., 94(A11), 15,321-15,333, doi:10.1029/JA094iA11p15321.

Emmert, A. D., D. P. Richmond, D. P. Drob (2010), A computationally compact representation of magnetic apex and quasi dipole coordinates with smooth base vectors. J. Geophys. Res. 115. doi:10.1029/2010JA015326.

Falkovich, G. (2008), Introduction to turbulence theory, in Cardy J., Falkovich, G., \&t Gawedzki, K. (Eds.), Non-Equilibrium Statistical Mechanics and Turbulence, Cambridge University Press.

Foster, J. C. (1993). Storm-time plasma transport at middle and high latitudes, J. Geophys. Res., 98,
1675-1689, doi: 10.1029/92JA02032.

Foster, J. C., et al. (2005). Multiradar observations of the polar tongue of ionization, J. Geophys. Res., 110, A09S31, doi:10.1029/2004JA010928.

Friis-Christensen, E., H. Lurh, and G. Hulot (2006), Swarm: a constellation to study the earth's magnetic field, Earth Planets Space, 58, 351

Frisch U. (1995), Turbulence. The legacy of A. N. Kolmogorov, Cambridge University Press, 179 p.

Golovchanskaya, I. V., A. A. Ostapenko, and B. V. Kozelov (2006). Relationship between the highlatitude electric and magnetic turbulence and the Birkeland field-aligned currents. J. Geophys. Res., 111, A12301.

Golovchanskaya, I. V., and B. V. Kozelov (2010). On the origin of electric turbulence in the polar cap ionosphere. J. Geophys. Res., 115, A09321.

Heppner, J. P., M. C. Liebrecht, N. C. Maynard, and R. F. Pfaff (1993). High-latitude distributions of plasma waves and spatial irregularities from DE2 alternating current field electric field observations. J. Geophys. Res., 98, 1629-1652.

Hurst, H. (1956). Methods of using long-term storage in reservoirs. ICE Proceedings, 5(704), 519-543.

Keeler S. P. and Y. Nievergelt (1998). Computing Geodetic Coordinates. SIAM Rev. 40, 2 (June 1998), 300309. Doi 10.1137/S0036144597323921.

Kelley, M. C., J. F. Vickrey, C. W. Carlson, and R. Torbert (1982), On the origin and spatial extent of highlatitude F region irregularities, J. Geophys Res., 87, 4469.

Keskinen, M. J., and S. L. Ossakow (1983), Theories of high-latitude ionospheric irregularities. A review, Radio Sci., 18(6), 1077-1091, doi:10.1029/RS018i006p01077.

Kintner Jr., P. M. (1976). Observations of velocity shear driven plasma turbulence. J. Geophys. Res., 81, 5114.

Knudsen, W. C. (1974). Magnetospheric convection and the high-latitude F2 ionosphere. J. Geophys. Res., 79, 1046-1055.

Knudsen, D. J., J. K. Burchill, S. C. Buchert, A. I. Eriksson, R. Gill, J.-E. Wahlund, L. Ahlen, M. Smith, and B. Moffat (2017), Thermal ion imagers and Langmuir probes in the Swarm electric field instruments, J. Geophys. Res. Space Physics, 122, doi:10.1002/2016JA022571.

Kozelov, B. V., I. V. Golovchanskaya, A. A. Ostapenko, and Y. V. Federenko (2008). Wavelet analysis of high latitude electric and magnetic fluctuations observed by Dynamic Explorer 2 satellite. J. Geophys. Res., 113, A03308. 
Laundal, K. M., and A. D. Richmond (2017), Magnetic coordinate systems, Space Sci Rev, 206: 27, doi: 10.1007/s11214-016-0275-y.

Yaqi, J., I. M. J.ran, and W. J. Miloch (2014), GPS scintillation effects associated with polar cap patches and substorm auroral activity: Direct comparison, J. Space Weather Space Clim., 4, A23, doi:10.1051/swsc/2014019.

Jin, Y., J. I. Moen, andW. J. Miloch (2015), On the collocation of the cusp aurora and the GPS phase scintillation: A statistical study, J. Geophys. Res. Space Physics, 120(10), 9176-9191, doi:10.1002/2015JA021449.

Oksavik, K., J. Moen, M. Lester, T. A. Bekkeng, and J. K. Bekkeng (2012), In situ measurements of plasma irregularity growth in the cusp ionosphere, J. Geophys. Res., 117(A11), A11301, doi:10.1029/2012JA017835.

Prikryl, P., P. T. Jayachandran, S. C. Mushini, and R. Chadwick (2011), Climatology of GPS phase scintillation and HF radar backscatter for the high-latitude ionosphere under solar minimum conditions, Ann. Geophys., 29, 377-392, doi:10.5194/angeo29-377-2011.

Pulkkinen, A., A. Klimas, D. Vassiliadis, V. Uritsky, E. and Tanskanen (2006). Spatiotemporal scaling properties of the ground geomagnetic field variations. J. Geophys. Res., 111, A03305.

Richmond, A. D. (1995), Ionospheric electrodynamics using magnetic apex coordinates, J. Geomag. Geoelectr., 47, 191- 212.

Ruiz-Chavarria, G., C. Baudet, and S. Ciliberto (1996), Scaling laws and dissipation scale of a passive scalar in fully developed turbulence, Physica D, 99, 369-380.

Sridhar, S., and P. Goldreich (1995). Toward a theory of interstellar turbulence: II. Strong Alfvenic turbulence, Astrophysics Journal, 438, 763-775.

Sugiura, M., N. C. Maynard, W. H. Farthing, J. P. Heppner, B. G. Ledley, and L. J. Cahill Jr. (1982). Initial results on the correlation between the magnetic and electric fields observed from DE 2 satellite. Geophys. Res. Lett., 9, 985-988.

Tsunoda, R. T. (1988), High-latitude F region irregularities: A review and synthesis, Rev. Geophys., 26(4), 719-760, doi:10.1029/RG026i004p00719.

Taylor, G. I. (1938). The spectrum of turbulence. Proc. R. Soc. London, 164(919), 476-490.

Villain, I.-P., C. Beghin, and C. Hanuise (1986), ARCAD3-SAFARI coordinated study of auroral and polar F region ionospheric irregularities, Ann. Geophys., 4, 61.
Xiong, C., C. Stolle, and H. Lühr (2016), The Swarm satellite loss of GPS signal and its relation to ionospheric plasma irregularities, Space Weather, 14, 563-577, doi: 10.1002/2016SW001439.

Xiong, C., C. Stolle, and J. Park (2018), Climatology of GPS signal loss observed by Swarm satellites, Ann. Geophys., 36, 679-693, https: //doi.org/10.5194/angeo-36-679-2018, 2018.
"CORRESPONDING AUTHOR: Fabio GIANNATTASIO, Istituto Nazionale di Geofisica e Vulcanologia, Rome, Italy email: fabio.giannattasio@ingv.it (C) 2019 the Istituto Nazionale di Geofisica e Vulcanologia. All rights reserved 